\title{
Implementation of the Chien search algorithm on a baseband processor
}

\author{
Youngseok Lee ${ }^{1}$, Hyunseok Lee ${ }^{1 \mathrm{a})}$, In-Young Jung ${ }^{1}$, and Ho Yang ${ }^{2}$ \\ ${ }^{1}$ Dept. of Electornics and Communications Engineering, Kwangwoon University, \\ 447-1 Wolgye-dong, Nowon-gu, Seoul 139-701, Republic of Korea \\ ${ }^{2}$ Samsung Advanced Institute of Science and Technology (SAIT), Mt 14-1, \\ Nongseo-dong, Giheung-gu, Yongin-si, Gyeonggi-do, 446-712, Republic of Korea \\ a) hyunseok@kw.ac.kr
}

Abstract: In this paper, we discuss the implementation of the $\mathrm{BCH}$ decoder Chien search algorithm on a SIMD style programmable baseband processor with minimum memory footprint and processing time degradation. Due to the emergence of long $\mathrm{BCH}$ codes, the computational efficiency of the Chien search algorithm becomes a major implementation issue for $\mathrm{BCH}$ decoders. We minimize the memory usage and processing time of the $\mathrm{BCH}$ decoders by deriving a computation rule, which is used to efficiently generate the power terms of primitive elements upon the SIMD datapath, instead of storing all pre-computed terms in the memory.

Keywords: programmable baseband processor, SIMD, BCH decoder, Chien search algorithm

Classification: Integrated circuits

\section{References}

[1] J. Cho and W. Sung, "Efficient software-based encoding and decoding of BCH codes," IEEE Trans. Comput., vol. 58, no. 7, pp. 878-889, July 2009.

[2] A. Kumar and K. Berkel, "Vectorization of reed solomon decoding of mapping on the EVP," Proc. Conf. Design Automation and Test in Europe, Munich, Germany, 2008.

[3] M. Moudgill, et al., "Galois field instructions in the Sandblaster 2.0 architecture," Int. J. Digital Multimedia Broadcasting, vol. 2009, Article ID 129698, 2009.

[4] A. Nohl, et al., "Application specific processor design," Int. Conf. Computer-Aided Design, San Jose, U.S.A., Nov. 2010. 


\section{Introduction}

Recently, the development of consumer devices having communication capabilities promotes the use of programmable processors for baseband signal processing tasks instead of application specific hardwired logics. This design approach will be adopted widely because of the flexibility, which allows for supporting multiple wireless communication standards and rapid time to market. However, the transition to this design style involves a challenging task due to tight budgets in power and processing time permitted by the baseband processors.

Previous studies on the architecture of programmable baseband processors show that the power and processing time requirements of the baseband signal processing workloads can be satisfied from low speed wireless terminals. Among many architectural schemes proposed in these studies, we adapted the single instruction multiple data (SIMD) scheme on our processor, hyperFLEX, for efficient processing of vector computations included in baseband signal processing operations. As shown in Fig. 1, it has both a 64-lane SIMD datapath for parallel workload and a scalar datapath for scalar workload. The parallel execution of 64 arithmetic units with a single control unit results in low power consumption and high throughput simultaneously.

In this paper, we discuss the implementation of Bose, Ray-Chaudhuri, and Hocquenghem $(\mathrm{BCH})$ decoder on the hyperFLEX, especially the decoder for the strong $\mathrm{BCH}$ code whose code length reaches several tens of kilobits. It is the first step of our research to implement a multifunctional channel decoder on a single programmable hardware platform, which is for various digital video broadcasting (DVB) systems such as DVB-terrestrial, cable, and satellite (DVB-T/C/S) and their next generations (DVB-T2/S2). Among the channel decoders of these DVB systems, the BCH decoder is one of the most challenging ones due to its high computational workload.

In this work, we found that the Chien search algorithm, which is to search exact error locations, dominates total workloads, especially in the decoding of long BCH codes. As shown in experiment section, the workload of the Chien search algorithm reaches about $40 \%$ of the total workload. In addition, the Chien search algorithm for the long $\mathrm{BCH}$ codes requires large memory, up to several hundred kilobytes, for storing the primitive element and its power terms. Even using the advanced silicon technology, it is challenging to implement this large memory in the form of fast on-chip memories. Thus, in this paper, we discuss how to implement the Chien search algorithm for long $\mathrm{BCH}$ codes on the hyperFLEX having a wide SIMD datapath, with reasonable memory footprint and processing time.

To achieve this goal, we process the vector computations existing in the Chien search computations with the 64-lane SIMD datapath of the hyperFLEX and reduce the size of the required memory by deriving a memory efficient computation rule of the primitive element and its power terms. Parallel processing of vector computations on the SIMD datapath improves not only the throughput but also the power efficiency of the $\mathrm{BCH}$ decoder by 
reducing operation clock speed. In addition, smaller memory enables us to realize the baseband processors on a smaller silicon die and it reduces implementation cost.

For validation, we implemented the hyperFLEX using a commercial processor design tool based on the language for instruction set architecture (LISA). From the LISA model, we generated hardware description language model such as Verilog and ported it on a field programmable gate array (FPGA). Then, the assembly program for realizing the Chien search algorithm was executed on the FPGA model with the assistance of the accompanied compilation tool chain which is also generated by the processor design tool.

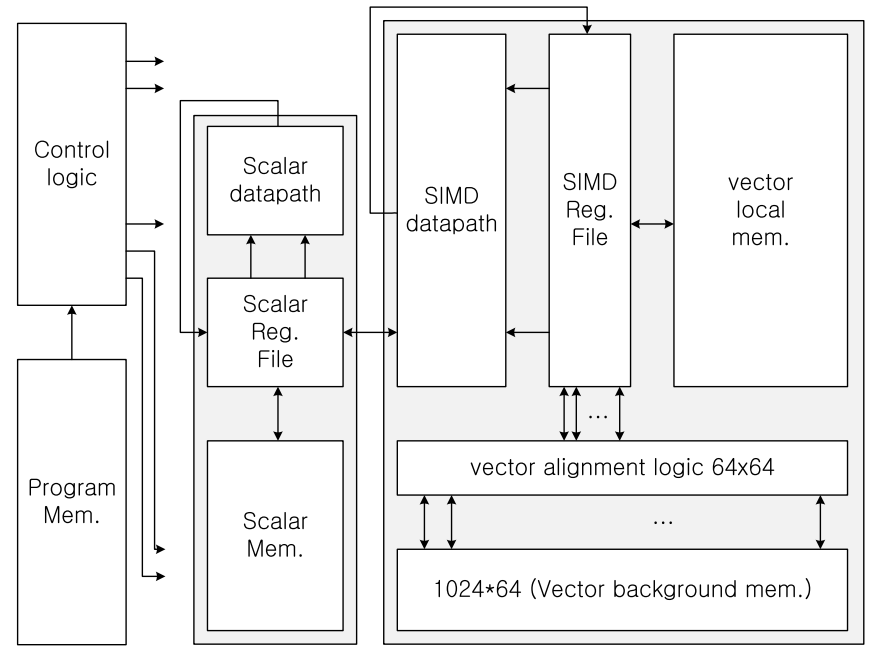

Fig. 1. Structure of the HyperFLEX; the wide SIMD baseband processor is used to implement the Chien search algorithm.

Numerous research results are available on the implementation of the $\mathrm{BCH}$ decoder on programmable processors. Cho's work [1] is limited to the case of using general purpose processors such as ARM or MIPS. In our case, it is possible to use application specific instructions to achieve better performance. NXP's EVP [2] only considers the BCH codes with short code length. In the case of a short $\mathrm{BCH}$ code, the memory space required for the corresponding Galois field primitive element and its power terms is not a critical design issue. Sandbridge's Sandblaster [3] does not address the decoder of long BCH codes, but instead, the efficient implementation of Galois multiplications in their baseband processor.

The rest of the paper is organized as follows. Section II explains fundamental issues with respect to the proposed scheme. Section III discusses the properties of the Chien search algorithm that are utilized for its efficient implementation over the SIMD datapath. The experiment results are presented in Section IV. Conclusions are drawn in Section V. 


\section{Preliminary}

\section{1 hyperFLEX}

As shown in Fig. 1, the hyperFLEX is a programmable baseband processor developed for the channel decoding operations of the DVB receivers. Thus, it can decode multiple channel codes such as Low Density Parity Check code, convolutional code, Reed-Solomon code, and $\mathrm{BCH}$ code without hardware changes. The reusability of such a hardware platform shortens the development time of a product.

The hyperFLEX processor has two datapaths. One is a 1024 bit (= 16 bits $\times 64$ lanes) wide SIMD datapath for vector workloads that can be efficiently represented by SIMD style instructions. The second is a 16-bit scalar datapath for sequential operations mixed with vector operations, for example loop control tasks. The SIMD datapath simultaneously achieves high throughput and low power consumption because of the parallel execution of an identical operation on all arithmetic units and the sharing of control logic between them, The hyperFLEX is a long-instruction word machine that embeds two independent sub-instructions for the scalar datapath and the SIMD datapath within an instruction. The parallel execution of these two sub-instructions further improves system throughput.

This processor has two register files and three memories. The scalar datapath is connected with a scalar register file and a scalar data memory. The scalar register file consists of 16 16-bit registers, one write port, and two read ports. The operation of the scalar datapath is almost identical to that of other conventional RISC processors except for having only minimal instructions required for implementing specific applications. The SIMD datapath has a SIMD register file and two vector memories, namely, the vector background memory and the vector local memory. The SIMD register file consists of 16 1024-bit registers, two read ports, and one write port. The vector background memory is exploited as an intermediate storage area for vector alignment operations. On the other hand, the vector local memory is used as a fast memory for storing vector data without vector realignment. As shown in Fig. 1, the SIMD register file and vector local memory are directly connected.

In this processor, the vector alignment operation is indirectly performed by a vector alignment logic that is placed between the SIMD register file and the vector background memory. For vector alignment operations, read or write operation with respect to the vector background memory is required. The vector alignment logic is a non-blocking switch that has 64 16-bit input ports and 64 16-bit output ports.

This processor has application specific instructions for a number of channel decoders; however, for succinctness, a detailed discussion on all instructions is not presented in this paper. One major difference of the instructions for the Chien search algorithm compared to other applications is the support of Galois field arithmetic in both the scalar datapath and the SIMD datapath. 


\subsection{BCH decoder}

The $\mathrm{BCH}$ decoder for these standards consists of three major blocks, namely, the syndrome generation block, the Berlekamp-Massey block, and the Chien search block. The syndrome generation block computes syndromes of the received code word. A Berlekamp-messy block is used to derive an error location polynomial from the syndromes generated in the previous step. Finally, the exact error locations of the received input data are determined by the Chien search block, which is to compute the output of the error location polynomial with respect to all input data bits.

The hyperFLEX, our SIMD-style baseband processor, is used to implement the $\mathrm{BCH}$ decoder. It is because the major computations of the $\mathrm{BCH}$ decoder can be represented as the inner products of two vectors as explained before, and thus can be optimally represented by SIMD instructions. Furthermore, the $\mathrm{BCH}$ decoder is well suited for wide SIMD datapaths because it only uses scalable hardware blocks. One representative non-scalable hardware block limiting the expansion of the width of SIMD datapath for higher throughput is the adder tree, which is used to add up all elements in a vector. For instance, the adder tree is used for the accumulation of multiplication results in a vector inner product. In the adder tree, the carry propagation time between adders, limits the maximum number of adders comprising it. However, in the Galois field underlying the $\mathrm{BCH}$ codes, additions can be presented as bit-wise exclusive OR operations that do not generate carry bits. Thus, in the $\mathrm{BCH}$ decoder, the adder is no more a limiting factor of the maximum SIMD width. The other non-scalable hardware block in programmable baseband processor is the shuffle network for the vector alignment. However, in the $\mathrm{BCH}$ decoder, no vector alignment operations are used. Thus, the shuffle network is not a limiting factor of the SIMD datapath scalability.

In addition, as shown in experiment section, the workload profile of the $\mathrm{BCH}$ decoder varies according to the change in the code length. Traditionally, due to the high computational loads, the $\mathrm{BCH}$ code was only adapted on short-length messages. Thanks to the progress in silicon process technology, recently, the $\mathrm{BCH}$ code has been deployed to protect long messages from errors. In long codeword $\mathrm{BCH}$ code, the fast processing of the Chien search and the syndrome computations are important due to their dominance in the total workload, which can reach up to $99 \%$.

\subsection{Chien search algorithm}

The Chien search algorithm is to compute (1) with respect to all input data locations, where $\mathrm{L}$ is the length of the received code word, $\nu$ is the maximum order of the error location polynomial, and $\alpha^{i}$ is the i-th power term of the primitive element $\alpha$ of the corresponding Galois field.

$$
\sigma\left(\alpha^{i}\right)=1+\sigma_{1} \alpha^{i}+\sigma_{2} \alpha^{2 i}+\cdots+\sigma_{v} \alpha^{v i},(0 \leq i<\mathrm{L})
$$

If we consider together these Chien computations performed on all bit positions from 0 to $\mathrm{L}-1$, (1) forms the matrix shown in (2). Throughout this 
paper, the notation, $\bar{\sigma}=\left[\sigma\left(\alpha^{0}\right), \sigma\left(\alpha^{1}\right), \cdots, \sigma\left(\alpha^{L-1}\right)\right]^{T}$, is named as the Chien result vector.

$$
\bar{\sigma}=\left[\begin{array}{cccc}
1 & 1 & \cdots & 1 \\
1 & \alpha^{1} & \cdots & \alpha^{v} \\
\vdots & \vdots & \ddots & \vdots \\
1 & \alpha^{L-1} & \cdots & \alpha^{(L-1) v}
\end{array}\right]\left[\begin{array}{c}
1 \\
\sigma_{1} \\
\vdots \\
\sigma_{v}
\end{array}\right]
$$

In the Chien search computations shown in (2), we can see that $(\nu+1) \times \mathrm{L}$ coefficients are required as input data, and $(\nu+1) \times \mathrm{L}$ Galois field multiplications are required for output generation. For instance, in a $\mathrm{BCH}$ code for the DVB systems, where $\mathrm{L}=54000$ and $\nu=12$, the memory space required for storing these coefficients is 1.4 Mbyte and the number of required Galois field multiplications is 702,000 per frame. The motivation for the derivation of the computation rules discussed in this paper is to minimized data memory required for decoding long $\mathrm{BCH}$ code.

\section{Efficient implementation of Chien search algorithm}

\subsection{Processing time enhancement}

As previously explained, the processing time of the Chien search algorithm is enhanced by mapping the Chien computations on the SIMD datapath of the hyperFLEX. This can be achieved because all Galois field multiplications shown in the Chien search algorithm have no data dependencies between them, as show in (2), and thus can be executed in parallel. There exist two possible approaches for the mapping the Chien computations on the SIMD datapath. One is to perform the computations in (2) on a row basis, and the other is on a column basis.

In a $\mathrm{BCH}$ code for DVB systems, since $\nu(=10$ or 12$)$ is smaller than the SIMD width $\mathrm{W}(=64)$ of the hyperFLEX, the row basis computation utilizes only $16 \% \sim 18 \%(=10 / 64$ or $12 / 64)$ of the arithmetic units of the SIMD datapath. Conversely, for the same DVB workload, the input data length L $(=54,000)$ is much greater than the $\mathrm{W}$, and thus the utilization of the SIMD datapath is near $100 \%$. Consequently, we perform all computations of the Chien search on a column basis.

In the column basis computation approach, (2) can be reformatted as shown in (3). In this equation, the $\otimes$ notation represents a Kronecker product operator, where $\left[\begin{array}{lllll}a_{0} & a_{1} & \ldots & a_{N-1}\end{array}\right]^{T} \otimes\left[\begin{array}{llll}b_{0} & b_{1} & \ldots & b_{N-1}\end{array}\right]^{T}=$ $\left[\begin{array}{llll}a_{0} b_{0} & a_{1} b_{1} & \ldots & a_{N-1} b_{N-1}\end{array}\right]^{T}$

$$
\bar{\sigma}=\left[\begin{array}{c}
1 \\
1 \\
\vdots \\
1
\end{array}\right] \otimes\left[\begin{array}{c}
1 \\
1 \\
\vdots \\
1
\end{array}\right]+\left[\begin{array}{c}
1 \\
\alpha^{1} \\
\vdots \\
\alpha^{L-1}
\end{array}\right] \otimes\left[\begin{array}{c}
\sigma_{1} \\
\sigma_{1} \\
\sigma_{1} \\
\sigma_{1}
\end{array}\right]+\cdots+\left[\begin{array}{c}
1 \\
\alpha^{1 v} \\
\vdots \\
\alpha^{(L-1) v}
\end{array}\right] \otimes\left[\begin{array}{c}
\sigma_{v} \\
\sigma_{v} \\
\vdots \\
\sigma_{v}
\end{array}\right]
$$

For succinctness, we represent this equation using two column vectors, $\bar{U}$ and $\bar{\alpha}_{i}$, where $\bar{U}=\left[\begin{array}{llll}1 & 1 & \cdots & 1\end{array}\right]^{T}$ and $\bar{\alpha}=\left[\begin{array}{llll}1 & \alpha^{1 \cdot i} & \cdots & \alpha^{(L-1) \cdot i}\end{array}\right]^{T}$.

$$
\bar{\sigma}=\bar{U} \otimes U+\bar{\alpha}_{1} \otimes\left(\sigma_{1} \cdot \bar{U}\right)+\cdots+\bar{\alpha}_{v} \otimes\left(\sigma_{v} \cdot \bar{U}\right)
$$


On the hyperFLEX, the Kronecker products in (4) are performed on the SIMD datapath. During computation, the $\sigma_{i} \cdot \bar{U}$ and $\bar{\alpha}_{i}$ terms are stored in the SIMD register file and provided as input operands.

Although the length of the Chien result vector, $L$, is greater than the $W$, we can compute $L$ Chien values by repeating the SIMD operations $k$ times, where $k=\lceil L / W\rceil$. If we defined Chien result subvector $\bar{\sigma}_{i}$ as the $i$-th portion of the Chien result vector $\bar{\sigma}$, then the Chien result vector $\bar{\sigma}$ can be divided into a number of Chien result subvectors ranging from $\bar{\sigma}_{0}$ to $\bar{\sigma}_{k}$ as shown in (5). Because no Chien result subvectors require other Chien result subvectors as their input data, it is possible to compute them in parallel.

$$
\bar{\sigma}=\left[\begin{array}{cccc}
{\left[\sigma\left(\alpha^{0}\right)\right.} & \sigma\left(\alpha^{1}\right) l & \cdots & \left.\sigma\left(\alpha^{W-1}\right)\right] \\
{\left[\sigma\left(\alpha^{W}\right)\right.} & \sigma\left(\alpha^{W+1}\right) & \cdots & \left.\sigma\left(\alpha^{2 W-1}\right)\right] \\
{\left[\sigma\left(\alpha^{(k-1) W}\right)\right.} & \sigma\left(\alpha^{(k-1) W+1}\right) & \cdots & \left.\left.\sigma\left(\alpha^{k W-1}\right)\right]\right]^{T}
\end{array}=\left[\begin{array}{c}
\bar{\sigma}_{0}^{T} \\
\bar{\sigma}_{1}^{T} \\
\vdots \\
\bar{\sigma}_{k-1}^{T}
\end{array}\right]\right.
$$

An additional property of computation pattern of the Chien search algorithm, which is exploited for reducing the processing time, is that, in most cases, a Galois field addition follows a Galois field multiplication. In the Galois field, an addition is a simple exclusive OR operation. Thus, the chaining of the addition with the multiplication so as to form a single instruction is viable with negligible implementation cost. Thus, the hyperFLEX has a gfmac instruction that performs a Galois field multiplication and an accumulation operation within one operation cycle.

\subsection{Minimization of local memory size}

The memory space required for storing the primitive element and its power terms used in the Chien search algorithm, $\bar{\alpha}_{1}$ to $\bar{\alpha}_{v}$ in (4), can be reduced by exploiting the relation between the primitive element and its power terms as shown in (6) where $1<i \leq \nu$.

$$
\bar{\alpha}_{i}=\left[\begin{array}{c}
1 \\
\alpha^{(i-1) 1} \\
\vdots \\
\alpha^{(i-1)(L-1)}
\end{array}\right] \otimes\left[\begin{array}{c}
1 \\
\alpha^{1} \\
\vdots \\
\alpha^{(L-1)}
\end{array}\right]=\bar{\alpha}_{i-1} \otimes \bar{\alpha}_{1}
$$

Equation (6) indicates that the primitive element vectors $\bar{\alpha}_{2}$ to $\bar{\alpha}_{v}$ can be generated from $\bar{\alpha}_{1}$. Thus, the size of the local memory required to store the primitive element and its power terms is reduced from $(L \times \nu)$ to $L$. Note that the length of $\bar{\alpha}_{1}$ is $L$. However, even the vector $\bar{\alpha}_{1}$ requires large amounts of memory for decoding long BCH codes, since $L$ could be 54,000 .

The local memory space required for the vector $\bar{\alpha}_{1}$ is further reduced by exploiting its other property observed between the $\bar{\alpha}_{1}$ subvectors, $\bar{\alpha}_{0,1}$ to $\bar{\alpha}_{k-1,1}$, whose length is equivalent to that of the SIMD datapath, $W$, as 


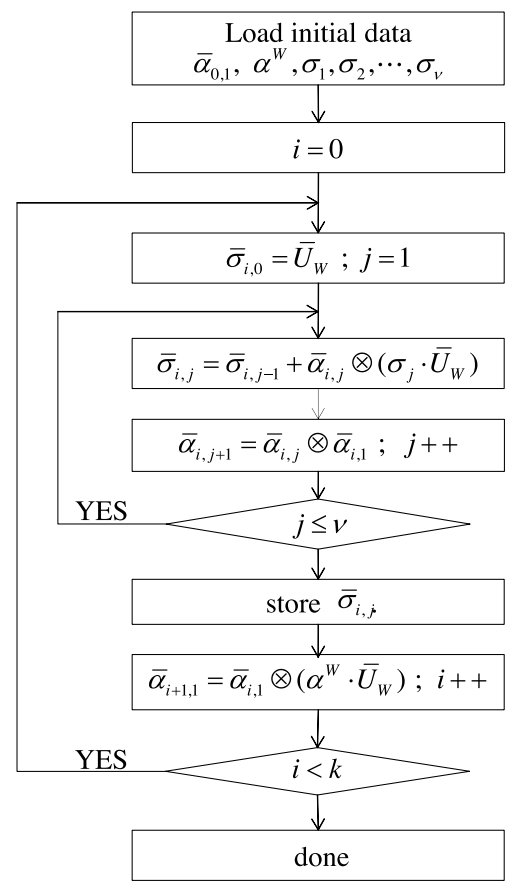

Fig. 2. The proposed computational procedure for the Chien search algorithm

shown in the following equation.

$$
\bar{\alpha}_{1}=\left[\begin{array}{cccc}
{[1} & \alpha^{1} & \cdots & \left.\alpha^{W-1}\right] \\
{\left[\alpha^{W}\right.} & \alpha^{W+1} & \cdots & \left.\alpha^{2 W-1}\right] \\
{\left[\alpha^{k W}\right.} & \left.\alpha^{k W+1}\right) & \cdots & \left.\left.\alpha^{k W+W-1}\right]\right]^{T}
\end{array}=\left[\begin{array}{c}
\bar{\alpha}_{0,1}^{T} \\
\bar{\alpha}_{1,1}^{T} \\
\vdots \\
\bar{\alpha}_{k-1,1}^{T}
\end{array}\right]\right.
$$

In addition, this equation leads to the following relation between the subvectors of $\bar{\alpha}_{1}$. Equation (8) shows that all the subvectors of $\bar{\alpha}_{1}$, from $\bar{\alpha}_{0,1}$ to $\bar{\alpha}_{k-1,1}$, can be generated only by $\bar{\alpha}_{0,1}$ and a scalar value $\alpha^{W}$.

$$
\bar{\alpha}_{i, 1}=\left[\begin{array}{c}
\alpha^{i W} \\
\alpha^{i W+1} \\
\vdots \\
\alpha^{i W+W-1}
\end{array}\right]=\left[\begin{array}{c}
\alpha^{(i-1) W} \\
\alpha^{(i-1) W+1} \\
\vdots \\
\alpha^{(i-1) W+W-1}
\end{array}\right] \otimes\left[\begin{array}{c}
\alpha^{W} \\
\alpha^{W} \\
\vdots \\
\alpha^{W}
\end{array}\right]=\bar{\alpha}_{i-1,1} \otimes \alpha^{W} \cdot \bar{U}
$$

Note that the length of $\bar{\alpha}_{0,1}$ is $W$ and the length of $\alpha^{W}$ is 1 . Thus, the total memory size required for storing the primitive element and its power terms is further reduced from $L$ to $W+1$. In the hyperFLEX, this amount of memory induces no memory overhead since $W$ is 64 .

\subsection{Implementation on the hyperFLEX}

By using the previous equations, we can implement the Chien search algorithm performed on the SIMD datapath of the hyperFLEX in a step by step basis as shown in Fig. 2. As proposed in this paper, the initial data $\bar{\alpha}_{0,1}, \alpha^{W}$ and $\sigma_{1}, \sigma_{2}, \cdots, \sigma_{v}$ are loaded from the scalar data memory and vector data memory of the hyperFLEX. 
First, we compute the first Chien result subvector, $\bar{\sigma}_{0}$, as shown in (9), where $\bar{U}_{W}$ is the unit column vector with length $W$. Equation (9) is the result of applying (5) upon (4). For generality, we explain the computation performed for the i-th Chien result subvector $\bar{\sigma}_{i}$.

$$
\bar{\sigma}_{i}=\bar{U}_{W}+\bar{\alpha}_{i, 1} \otimes\left(\sigma_{1} \cdot \bar{U}_{W}\right)+\cdots+\bar{\alpha}_{i, v} \otimes\left(\sigma_{v} \cdot \bar{U}_{W}\right)
$$

While computing the output of (9), each term $\bar{\alpha}_{i, j}$, where $0<j \leq v$, is computed from $\bar{\alpha}_{i, j-1}$ and $\bar{\alpha}_{i, 1}$ by exploiting the rule shown in (6) and (7).

The computations shown in (9) are iteratively performed by using the gfmac instruction of the hyper FLEX as shown in (10). In equation (10), $\bar{\sigma}_{i, j}$ is the $\mathrm{j}$-th partial computation result of the $\bar{\sigma}_{i}$. Thus, we need to store the last term $\bar{\sigma}_{i, v}$ as an operation result.

$$
\bar{\sigma}_{i, j}=\bar{\sigma}_{i, j-1}+\bar{\alpha}_{i, j} \otimes\left(\sigma_{j} \cdot \bar{U}_{W}\right)
$$

In the next step, we compute the next Chien result subvector, $\bar{\sigma}_{i+1}$. In this point, we derive $\bar{\alpha}_{i+1,1}$ from the $\bar{\alpha}_{i, 1}$ by exploiting the rule shown in (8). Then, the identical procedure is repeated until the last partial Chien result vector, $\bar{\sigma}_{k-1}$, is achieved. Note that in the proposed scheme no data memory is required for storing intermediate operation results except for initial data.

\section{Experimental results}

\subsection{Experimental environment}

For experimentation purposes, we implemented the hyperFLEX with a Synopsys' Processor Designer tool [4] that is based on LISA. The LISA model of the hyperFLEX was automatically converted into a Verilog model, and then ported on a Xilinx's Virtex-5 FPGA. A compilation tool chain was also generated from the Processor Designer and was used for the generation of executable codes.

Fig. 3 shows our test environment consisting of a Virtex-5 FPGA, a JTAG for FPGA, a serial peripheral interface (SPI) module, and a laptop computer. The SPI module is used for accessing the program and data memory of the hyperFLEX from the connected laptop computer. With this tool, we can download the application programs of the hyperFLEX, write initial data into the data memory, control its operation and read operation results from the data memory.

\subsection{BCH decoder workload profile}

Fig. 4 shows the variation of the $\mathrm{BCH}$ decoder workload profile with respect to code length change. The workload percentile is measured by executing $\mathrm{BCH}$ decoders on a Linux machine and counting the required operation cycles. In order to investigate the impact of the length of $\mathrm{BCH}$ codes on the total workload profile, we selected three representative $\mathrm{BCH}$ decoders with different code length: 48 bits, 2,040 bits, and 16,383 bits. Code1 is a binary $(48,36) \mathrm{BCH}$ code used in the control channels of cellular TDMA wireless communications systems. Code2 is a binary $(2,040,1,930) \mathrm{BCH}$ code used in 


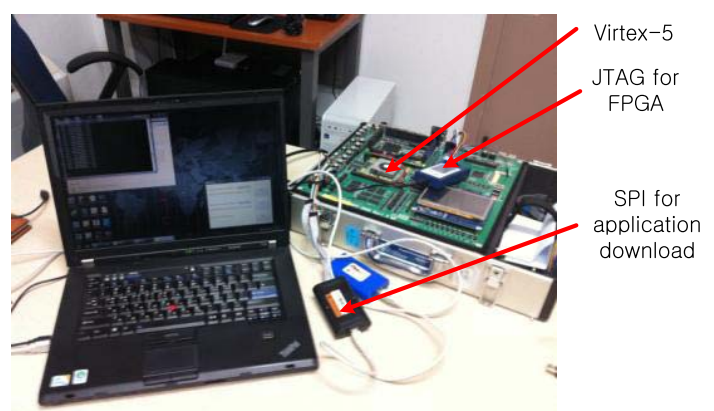

Fig. 3. Test environment used for implementing the hyperFLEX

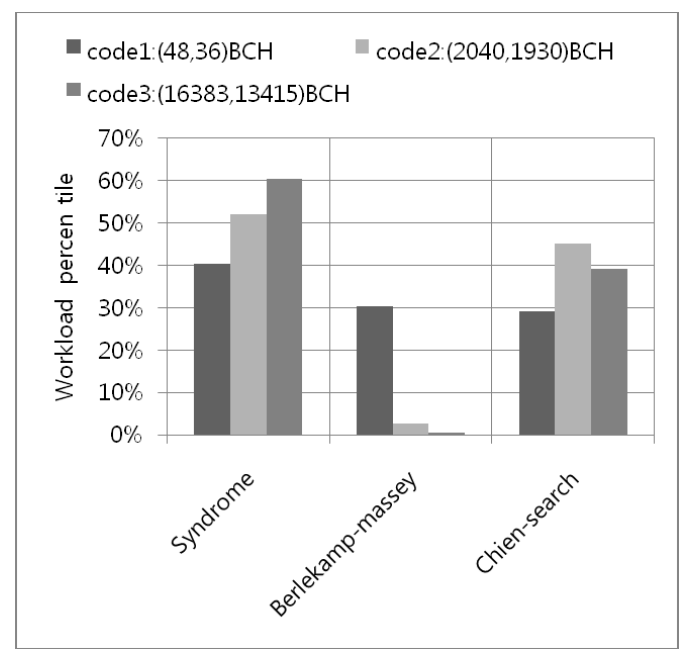

Fig. 4. The BCH decoder workload variation profile, according to the code length variation

the ITU-T G.975.1 optical fiber submarine cable systems. Code3 is a binary $(16,383,13,415)$ BCH code used in the DVB-S2 systems.

In the case of the short $\mathrm{BCH}$ codes (code1), three algorithm blocks almost evenly dominate total workload (syndrome 40\%, Berlekamp-Massey 30\%, and Chien search 30\%). However, as the code length increases, the Chien search and syndrome blocks dominate the total workloads (as seen by code2 and code3). This result is due to the fact that the workload of the BerlekampMassey block is linearly proportional to the number of maximum correctable error bits, which in general is not sensitive to the code length variation. The experimental results demonstrate that efficient processing of the Chien searching algorithm is also crucial in implementing the decoders for long $\mathrm{BCH}$ codes.

\section{3 hyperFLEX memory profile and throughput}

For evaluation purposes, we implemented the Chien search algorithm for the $(16,383,13,415)$ BCH code in two forms, namely, model-1 and model-2. Model-1 is an optimized version that exploits the properties of the primitive element and its power terms discussed in this paper. The operation of model2 is based on an assumption that the pre-computed primitive element and all 
Table I. Memory profile and throughput of the hyperFLEX

Model-1(opt.) Model-2(w/o opt.)

\begin{tabular}{lcc} 
Throughput(Kcycles) & 29.7 & 28.8 \\
Local memory (Kbytes) & 5 & 393 \\
\hline
\end{tabular}

its power terms are stored in advance in the local memory of the hyperFLEX.

As shown in Table II, the model-1 memory profile is approximately 80 times smaller than that of the model-2 thanks to exploiting to the computation rule derived in this paper. Although the model-1 spends a part of its operation time for computing the primitive element vector according to the property shown in (6) in run time, its total processing time is almost identical to that of the model-2. It is because of the long memory access time of the hyperFLEX. Note that, in the hyperFLEX, we use slow multi-cycle memories to save power, and, in model-2, the pre-computed primitive element and its power terms are stored in the slow data memory.

In conclusion, the experiment results show that the proposed implementation scheme for the Chien search algorithm successfully reduces the required memory size with slight degradation on the processing time of the programmable baseband processor.

\section{Conclusion}

In this paper, we proposed a cost-effective implementation technique for the Chien search algorithm on a programmable baseband processor having a wide SIMD datapath. We reformatted the Chien computations for efficient processing in the SIMD datapath. We successfully improved the processing time of the processor by utilizing the 64-lane SIMD datapath and minimized the local memory size for the primitive element and its power terms by applying the derived parameter generation rules. The proposed technique is validated on the FPGA evaluation board, which shows memory reduction by 80 times with only $5 \%$ performance degradation.

\section{Acknowledgments}

This work was supported in part by Research Grants from Kwangwoon University, Korea, in 2010 and Samsung Advanced Institute of Technology (SAIT), Korea. 